\title{
Spinal cord ischemia-reperfusion injury: MicroRNAs and mitophagy at a crossroads
}

\author{
Leora B. Balsam, MD
}

\author{
From the Department of Cardiothoracic Surgery, New York University-Langone Medical Center, New York, NY. \\ Disclosures: Author has nothing to disclose with regard to commercial support. \\ Received for publication May 31, 2017; accepted for publication June 5, 2017; available ahead of print June 30, \\ 2017. \\ Address for reprints: Leora B. Balsam, MD, Department of Cardiothoracic Surgery, NYU-Langone Medical Cen- \\ ter, 530 First Ave Suite 9V, New York, NY 10016 (E-mail: leora.balsam@nyumc.org). \\ J Thorac Cardiovasc Surg 2017;154:1509-10 \\ $0022-5223 / \$ 36.00$ \\ Copyright (C) 2017 by The American Association for Thoracic Surgery \\ http://dx.doi.org/10.1016/j.jtcvs.2017.06.010
}

Paralysis is a dreaded complication of descending and thoracoabdominal aortic reconstruction. Its occurrence is influenced by 2 distinct phases of spinal cord injury. The first is ischemia, which occurs during a period of aortic crossclamping or circulatory arrest. The second phase is reperfusion, which results in release of deleterious reactive oxygen species, inflammation, and additional cell death. Cell death after spinal cord ischemia-reperfusion occurs via 3 pathways: necrosis, apoptosis, and autophagic cell death. ${ }^{1}$ Strategies to mitigate spinal cord injury after ischemia-reperfusion would have obvious clinical utility to the cardiac surgeon, because our current tools (including cerebrospinal fluid drainage, distal aortic perfusion, and hypothermia) are limited in their ability to reliably protect against spinal cord injury. In this issue of the Journal, Liu and colleagues ${ }^{2}$ investigate the intersecting roles of microRNA-124 (miR-124) and mitophagy after spinal cord injury.

Several experimental models have been developed to study the cellular and molecular events after spinal cord injury. Rodent models include spinal cord crush or contusion, which models traumatic spinal cord injury, and transient aortic crossclamping, which models spinal cord ischemia-reperfusion. In all of these models, a common feature of the secondary injury phase is induction of neuronal autophagy. ${ }^{1,3-5}$ Autophagy is an evolutionarily conserved catabolic process for removing unwanted cytosolic proteins and damaged organelles through the autophagosome/lysosomal pathway. Autophagy is upregulated during conditions of nutrient deprivation or increased oxidative stress (eg, spinal cord ischemiareperfusion). It can have a cytoprotective function but also can result in excessive self-digestion and autophagic cell death (a type of programmed cell death). How that balance is maintained is poorly understood. A selective autophagic process for mitochondria, mitophagy, regulates mitochondrial number in response to metabolic demand and eliminates damaged mitochondria. In conditions of stress, mitophagy may protect the cell against release of mitochondrial contents, including reactive oxygen species, but it also

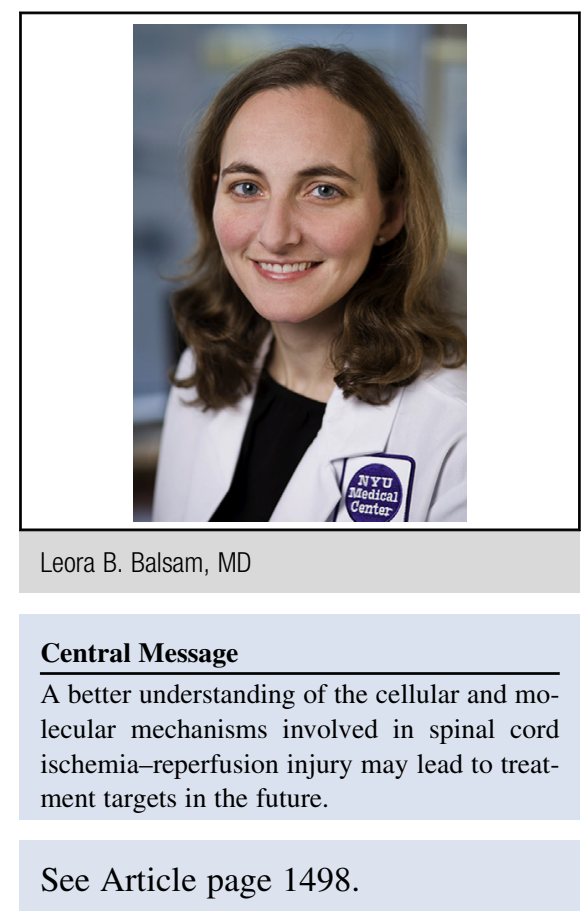

may result in excessive mitochondrial clearance, limiting cellular energy production. The specific role of mitophagy after spinal cord injury has received little attention, and Liu and colleagues ${ }^{2}$ postulate that increased mitophagy will be protective after spinal cord ischemia-reperfusion.

The molecular response to spinal cord injury also includes temporal and regional changes in gene expression in the spinal cord. miRNAs are an important class of small noncoding RNAs that post-transcriptionally regulate gene expression. Several miRNAs are specific to or enriched in the central nervous system, including miR-124. miR-124, the most abundant miRNA in the adult mammalian brain, plays a key role in neuronal differentiation. Lui and colleagues ${ }^{2}$ hypothesize that downregulation of miR-124 in rats with spinal cord ischemia-reperfusion will protect against spinal cord injury. In their model, spinal cord miR-124 expression is modulated by intrathecal transfection with a lentiviral vector encoding an miR-124 antagonist (antagomiR-124). Five days later, spinal cord ischemia-reperfusion is caused by transient descending aortic crossclamping. The authors find efficient downregulation of miR-124 expression in the animals pretreated with antagomiR-124 lentiviral vector. Neurologic function, as measured by the Motor Deficit Index score, was significantly improved compared with animals treated with 
control lentiviral vector or vehicle. This finding suggests that downregulation of miR-124 expression may have therapeutic benefit after spinal cord ischemia-reperfusion.

The secondary aim of Lui and colleagues ${ }^{2}$ is to evaluate whether altered mitophagy is a pathway through which miR-124 downregulation protects the spinal cord after ischemia-reperfusion. By using transmission electron microscopy for ultrastructural analysis (the gold standard methodology for assessing mitophagy), as well as direct measurements of markers of autophagy (Beclin-1 and LC3-II) from the mitochondrial fraction, the authors find evidence that mitophagy is increased after transfection with antagomiR-124 lentiviral vector. This increase in mitophagy is selectively blocked by 3-methyladenine (3-MA), an inhibitor of autophagy. Moreover, treatment with 3-MA abrogates much (although not all) of the beneficial antagomiR-124 effect on neurologic function. Taken together, the authors conclude that miR-124 downregulation results in increased mitophagy, which is protective after spinal cord ischemia-reperfusion.

Modifying cellular processes and molecular pathways to prevent spinal cord ischemia-reperfusion injury may not be so simple clinically. Inferences drawn from experimental animal models may not carry over to human systems. Moreover, modulating expression of miRNAs may have unwanted side effects that are not identified in a short-term animal model. Notably, miR-124 is postulated to regulate more than 1000 genes in the central nervous system, so treatment with antagomiR-124 likely will result in some off-target effects. Also, the timing of the treatment may matter. Fang and colleagues ${ }^{4}$ showed that modulating autophagy with 3-MA after spinal cord crush injury in rats was protective against motor neuron loss and apoptosis when given immediately after spinal cord injury, but it was detrimental when administered 24 hours later. Wei and colleagues $^{5}$ found that intrathecal injection of 3-MA after spinal cord ischemia-reperfusion injury resulted in improved neurologic function, a finding that was not reproduced by Liu and colleagues. ${ }^{2}$ Small differences in the models, timing of therapies, and drug-delivery modes may explain the divergent results.

Finally, it is worth noting that others have found miR-124 downregulation and mitophagy induction after spinal cord injury, even in the absence of treatment with antagomiR124. Zhao and colleagues ${ }^{6}$ demonstrated neuronal loss of miR-124 expression in the perilesion area in mice with spinal cord crush injury, and Nakanishi and colleagues ${ }^{7}$ found decreased miR-124 expression 1 to 7 days after spinal cord compression injury in mice. Yu and colleagues ${ }^{1}$ reported induction of neuronal mitophagy after spinal cord contusion in rats. Placed in the context of prior work, the strategy taken by Liu and colleagues ${ }^{2}$ seems to amplify normal response-to-injury mechanisms that occur after spinal cord injury.

\section{References}

1. Yu D, Li M, Ni B, Kong J, Zhang Z. Induction of neuronal mitophagy in acute spinal cord injury in rats. Neurotox Res. 2013;24:512-22.

2. Liu K, Yan L, Jiang X, Yu Y, Liu H, Gu T, et al. Inhibition of microRNA-124 protects against spinal cord ischemia-reperfusion injury partially through a mitophagy-dependent pathway. J Thorac Cardiovasc Surg. 2017; 154:1498-508.

3. Kanno H, Ozawa H, Sekiguchi A, Yamaya S, Itoi E. Induction of autophagy and autophagic cell death in damaged neural tissue after acute spinal cord injury in mice. Spine. 2011;36:E1427-34.

4. Fang B, Li X, Bao N, Tan W, Chen F, Pi X, et al. Role of autophagy in the bimodal stage after spinal cord ischemia reperfusion injury in rats. Neuroscience. 2016; 328:107-16.

5. Wei X, Zhou Z, Li L, Gu J, Wang C, Xu F, et al. Intrathecal injection of 3-methyladenine reduces neuronal damage and promotes functional recovery via authophagy attenuation after spinal cord ischemia/reperfusion injury in rats. Biol Pharm Bull. 2016;39:665-73.

6. Zhao Y, Zhang H, Zhang D, Yu C, Zhao X, Liu F, et al. Loss of microRNA-124 expression in neurons in the peri-lesion area in mice with spinal cord injury. Neural Regen Res. 2015;10:1147-52.

7. Nakanishi K, Nakasa T, Tanaka N, Ishikawa M, Yamada K, Yamasaki K, et al. Responses of microRNAs $124 \mathrm{a}$ and 223 following spinal cord injury in mice. Spinal Cord. 2010;48:192-6. 